\title{
Effect of Solid-State Laser Parameters on the Surface's Topography Formation During Texturization of Hard Metal Cutting Tools
}

\author{
Patrícia Helena de Oliveira Teixeira ${ }^{1,2}$, Adriano Gonçalves dos Reis ${ }^{1,2}$, Andreas Janssen ${ }^{3}$
}

\begin{abstract}
The objective of this paper is to analyze the influence of specific laser system parameters on the microstructure of the ring formation process on the hard metal tool's surface. The parameters analyzed were: working distance, incident beam power and tool surface treatment, flank and rake. The microstructures were analyzed with regard to its diameter and depth by an optical 3-D surface metrology instrument. The analyses were made by comparing the ring's process formation with the dimples' process formation, a well-known process called ablation. The results show that the ring's diameter is increased by increasing incident beam power or the distance of the laser beam's focus for both surfaces. In addition, increasing the working distance decreases the ring's depth for both surfaces.
\end{abstract}

KEYWORDS: Surface finishing, Pulsed lasers, Ablation, Microstructure.

\section{INTRODUCTION}

One of the biggest concerns of industry is the improvement of manufacturing efficiency in order to obtain minimization of production costs, maximization of production rate and maximization of production accuracy, etc.. In aeronautic industry, these are main concerns as well, especially with the prediction that the number of new airplanes will grow by more than 27.000 until 2025 compared to 2005 (Klocke et al., 2012). This prediction leads to an economic pressure on the aviation industry manufacturers, and the search for optimization in the manufacturing processes becomes necessary.

For the aircraft components construction, several manufacturing processes are used, and machining stands between the most applied ones. According to Aurich et al. (2011), during machining process, high mechanical and thermal loads occur at the cutting tool, causing tool failure. Due to this, optimization at the cutting tool represents an advantageous possibility. Cutting tools have been optimized by improvements of cutting tool material, coating system and tool macrogeometry. however, cutting tools with higher qualities regarding material removal rates, chip removal of hard-to-machine materials and tool life are each day more requested.

1.Faculdade de Tecnologia de São José dos Campos - São José dos Campos/SP - Brazil 2.Instituto Tecnológico de Aeronáutica - São José dos Campos/SP - Brazi 3.Fraunhofer Institute for Production Technology - Aachen - Germany. 


\section{LASER-BASED CUTTING TOOL PRODUCTION}

Looking on technical and mechanical assemblies, contact surfaces mainly determine the performance of systems in terms of being the interfaces where energy is transferred, converted and dissipated. The preparation of cutting tools using laser radiation is a useful improvement compared to the use of conventional techniques for optimization of these surfaces. Laser processing is a very powerful technique for manufacturing modification of defined cutting tool geometries. It is a non-contacting, wear free technique and not only offers highest flexibility due to the fact that the beam can be moved very fast by optical devices, but also provides excellent control of shape and size of microstructures, and, furthermore, a process relatively clean to the environment (Etsion, 2004).

According to Aurich et al. (2011), certain morphology modifications of contacted surfaces on cutting tools can improve tool performance. The so called laser-manufactured "micro-dimples" are microstructures already analyzed in recent research (Scaraggi et al., 2013; Kim et al., 2012). The fundamental formation process is the well-known process of laser ablation, which forms single ablation cavities by single laser pulses or complex superposition of many pulses.

According to Meijer et al. (2002), the term "ablation" relates to the effect of vaporizing irradiated material by incident laser beam. According to Poprawe (2004), laser radiation is used as a working tool to structure matter by ablation, defining the lateral precision. For this reason, laser radiation is projected by objectives onto the surface, thereby being formed to a specific spatial intensity distribution. Ablation is a process strongly dependent of pulse duration and wavelength, and it can occur by two main principles: short pulse and ultra-short pulse laser ablation. The type of ablation process determines the finishing quality of the ring (Poprawe, 2004). The characteristic of the ablation process - short or ultra-short laser ablation - is determined by the pulse duration, as shown in Fig. 1.

When the pulse duration is longer than 1 nanosecond ( $1 \mathrm{~ns}=10^{-9} \mathrm{~s}$ ), the process is called short pulse laser ablation and its major characteristic is the significant amount of material being removed from the irradiated area in liquid phase. At this process, there is a high absorption of laser energy in the vaporized material along the duration of one pulse. When the pulse duration is smaller than $1 \mathrm{~ns}$, the heating mostly takes place after the period of time of pulse duration and the process is called ultra-short pulsed laser. In opposite to pure ablation process, the formation process of rings is still not widely studied. During the rings' formation, there is just a slightly melting of surface material and very few of it is evaporated. According to Cabeza et al. (2012), the morphology at the surface can be characterized as solidified melted zone combining fine grain appearance and waves in the radial direction.

Janssen (2012) introduced the possible benefits of such microstructures manufactured by laser ablation on highly stressed parts. Based on this, in the present context, the functionalizing aims of three technological benefits for the cutting process are:

- An optimized surface topography enables the reduction of friction and cutting forces in the regime of direct tool-work piece contact.

- The lubrication shall be improved, leading then, to a reduction of tool's wear and hence to increased tool's life time. Alternatively higher cutting velocities can be applied without decreased life time.

- For several applications, it can be assumed that the current laser process allows supplying a higher surface quality on the treated work piece and thus increases an qualitative process output.

These benefits can be achieved optimizing tool surface by the combination of two phenomena: formation of a beneficial topography and formation of a new material microstructure in a surface near a layer of several hundred nanometers. The target surfaces are the flank and rake surface of cutting tools.

The target surface's texture in the present work is called "rings structure". Figure 2 shows an example of both structures,

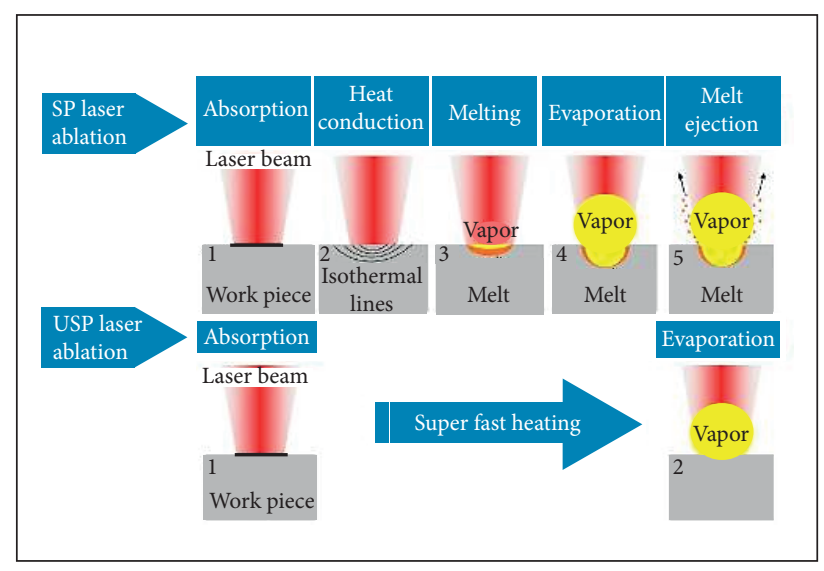

Figure 1. Physical mechanisms in SP and USP laser ablation (Janssen, 2012). 


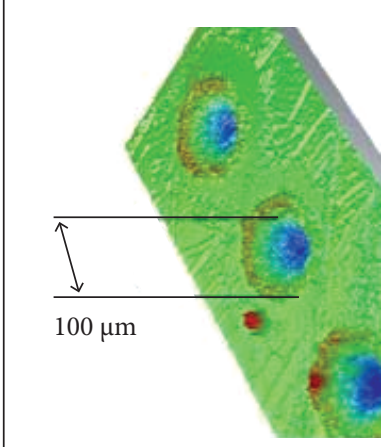

(a)

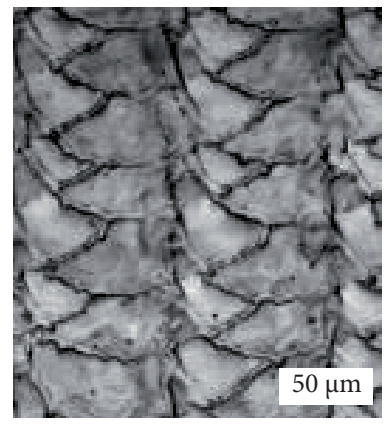

(b)
Figure 2. (a) regular dimples, distributed on the surface, and (b) textured composed of overlapping dimples, completely covering the surface (Klocke and Schmitt, 2012).

dimples and rings. The difference between both microstructure is that, unlike dimples, which allow some parts of the original tool material to be in contact with the workpiece, in the ring's microstructure, the tool surface in contact with the workpiece is completely modified by laser texturing. The present work aimed analyzing the formation process of ring's microstructure subjected to the main process parameters.

Due to positive results considering tool life, high material removal rates etc., obtained in previous works, the laser texturing process becomes an important tool in the cutting tool preparation field. Research about the laser functions and operation methods is necessary in order to define a behavior profile of laser parameters in obtaining textured surfaces, especially when these textured surfaces are now starting to be used, as for the case of ring's structures.

\section{METHODOLOGY - STUDY ON RING'S FORMATION}

The work was performed at the Fraunhofer Institute for Production Technology (IPT), a research laboratory, localized in the city of Aachen, Germany. For texturing experiments, a custom-designed laser structuring machine has been utilized, which was installed within former research projects.

\section{EXPERIMENTAL SETUP}

Based on industrial as well as research demands, the machine has been designed based on a 5-axis milling machine, which was adapted by implementation of an entire nanosecond laser ablation system including laser source, transportation fiber and laser scanner. With this machine, a real 3-D laser structuring process is possible by means of using 8 axes (5 machine, 3 optical, 1 virtual).

The laser source used was the Ytterbium pulsed fiber laser YLPM-1-A4-20-20, from IPG Laser. Regarding to its configurations, the laser presents an extended pulse repetition rate from 1.6 to $1,000 \mathrm{kHz}$, selectable pulse durations $(4,8,14$, $20,30,50,100$ and $200 \mathrm{~ns}$ ) and maximum peak power of $15 \mathrm{~kW}$.

The tool material used was cemented carbide from the group $\mathrm{K}$ (red), a conventional tungsten carbide grade. The material was chosen by the fact that the cemented carbide is one of the most utilized cutting tool materials today and the group derived from tungsten carbide is most readily employed.

The tool utilized was manufactured by SECO, from the model TNMA160404F, 410, 5 $\mu$. It was specially developed for specific applications in Fraunhofer IPT. It presents the flank surface specially treated but the rake surface is free of any surface treatment, which means the laser hits a hard metal WC-Co surface.

\section{EXPERIMENTAL APPROACH}

It has to be considered that the formation of the desired rings is based on a reduced ablation process. The process parameters are selected for causing a slight melt formation on the surface and subsequent evaporation of a very small amount of heated material. In regular laser ablation, this amount is decreased to the achievable maximum, since it enables high material removal and thus ablation rate. Here, the evaporation is only utilized to evolve a back pressure on the molten material. The molten layer, however, is only several hundred nanometers in thickness, thus the re-solidification takes place very fast, leading to the formation of rings in terms of "frozen liquid structures". For a better understanding, it can be imagined a stone being thrown in the water, and then it immediately solidifies in the first ring's formation.

Among the existing process parameters, which define a laser process, this work focused on the influence of two main process parameters: laser power and working distance. These process parameters control the process. However, they are no real process quantities. In the following, the meaning of these two parameters is described.

The laser power $\mathrm{P}_{\mathrm{L}}$ describes the average laser radiation energy emitted by the laser source per time. Pulsed laser emits pulses with single durations much smaller than $1 \mathrm{~s}$. In this case, the size of average output power of the laser is sometimes misleading, since it includes the time between the pulses. In the process of SP and USP laser processing, the pulse pause, which separates 
each pulse from the following one is commonly several thousand times higher than the pulse duration. Thus, the average power $\mathrm{P}_{\mathrm{L}}$ can be several ten Watts only, while the pulse peak power $\mathrm{P}_{L M}$, which is the absolute power within the pulse duration, can reach many thousand Watts.

To meet this fact, it is common in the field of SP and USP laser ablation processing to give not the laser output power but the intensity I or the fluency F, while the intensity gives the energy coupled into a unit area per time (Eq. 1):

$$
I=\frac{E_{p}}{A \cdot t_{L}}
$$

where $E_{P}$ is the pulse energy; $\mathrm{A}$ is the focal spot area and $t_{L}$ is the pulse duration.

The fluency gives the energy coupled into a unit area without time information, according to Eq. 2.

$$
F=\frac{E_{p}}{A}
$$

This is more common in USP laser processing, where effects can be interpreted independently from the pulse duration. In the project around this work, a laser has been utilized not only in the SP regime but it is additionally capable of emitting pulses of variable pulse duration. The pulses can be selected between 4 and 200 ns. Based on preliminary investigations, the pulse duration was kept constant at 100 ns.

In the present work's evaluations, the parameter $\mathrm{P}_{\mathrm{L}}$ is given. So, the variables $A$ and $E_{P}$ could be varied independently.

The working distance is the distance between focusing lens and work piece. To understand the meaning, Fig. 3 shows the beam propagation of a laser beam. By varying the working distance $z_{W}$, the spot diameter can be adjusted, offering the possibility to control the focal spot size. This is limited due to the laser beam's propagation profile, which is the intensity distribution within a fixed $z$-plane.

Having these two control parameters, laser power and working distance, one is able to influence the ring formation in terms of varying the irradiated and the impinged energy per area. The main characteristics which are evaluated concerning the ring's formation are their diameter and depth. The arrangement of rings in a pattern can be easily done by adapting the program which controls the tool path for the laser scanner mirrors and thus is not investigated in this work.

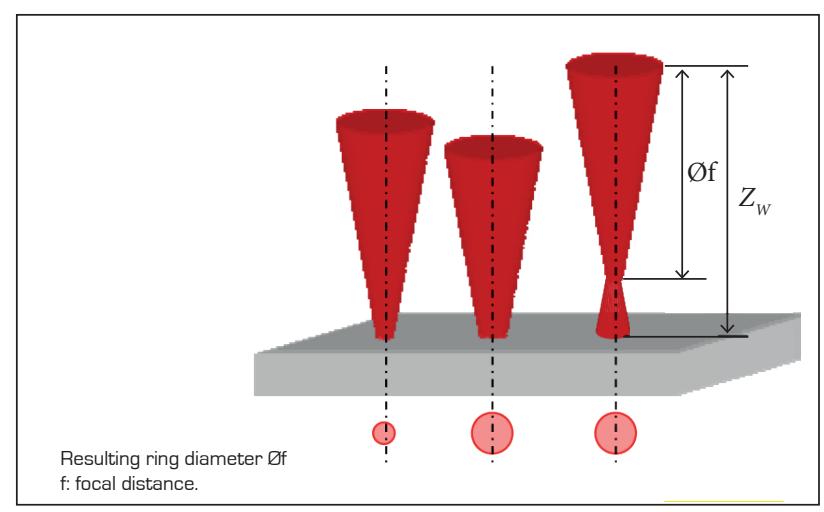

Figure 3. Beam propagation and spot size variation [Janssen, 2012).

Because the rake and the flank surfaces passed by different treatments, each surface has, therefore, different properties. For this reason, both were laser-treated and the laser parameters' influence was also investigated under the circumstances of the two material differences.

The surface measurements - diameter and depth - of the structured tool were made by the surface measurement device Alicona Infinite Focus Real 3-D - an optical 3-D surface metrology instrument. It automatically measures the complete structure of a surface (form, roughness and contour, for example).

For the tests, 20 different laser parameter combinations were defined, as shown in Table 1, in order to achieve different diameters and depth in the rings' structure.

\section{RESULTS AND DISCUSSION DIAMETER ANALYSIS}

The first results were obtained by varying the power percentage. The working distance was kept in $-0.8 \mathrm{~mm}$. The graph from Fig. 4 shows the diameter's behavior through the variation of the power percentage. The increment in diameter presents a tendency of growth when the incident beam power is increased. In the flank surface, which received the special surface treatment, the increment in the diameter is constant and it occurs in a smoother behavior. On the other hand, for the rake surface, where there is no treatment, the increasing in the diameter occurs through an irregular manner.

In the second analysis, the power was kept constant at $100 \%$, while the working distance was varied. The graph from Fig. 5 shows a comparison of the diameter's behavior between the two surfaces tested. 
Table 1. Laser parameter variation.

\begin{tabular}{|c|c|c|c|}
\hline Parameter & $\begin{array}{c}\text { Working distance } \\
\text { (mm) }\end{array}$ & Power [\%] & Tool surface \\
\hline 1 & -0.4 & 100 & Rake \\
\hline 2 & -0.7 & 60 & Rake \\
\hline 3 & -0.7 & 80 & Rake \\
\hline 4 & -0.7 & 100 & Rake \\
\hline 5 & -0.8 & 70 & Rake \\
\hline 6 & -0.8 & 80 & Rake \\
\hline 7 & -0.8 & 90 & Rake \\
\hline 8 & -0.8 & 100 & Rake \\
\hline 9 & -0.8 & 50 & Rake \\
\hline 10 & -0.8 & 60 & Rake \\
\hline 11 & -0.4 & 100 & Flank \\
\hline 12 & -0.7 & 60 & Flank \\
\hline 13 & -0.7 & 80 & Flank \\
\hline 14 & -0.7 & 100 & Flank \\
\hline 15 & -0.8 & 70 & Flank \\
\hline 16 & -0.8 & 80 & Flank \\
\hline 17 & -0.8 & 90 & Flank \\
\hline 18 & -0.8 & 100 & Flank \\
\hline 19 & -0.8 & 50 & Flank \\
\hline 20 & -0.8 & 60 & Flank \\
\hline
\end{tabular}

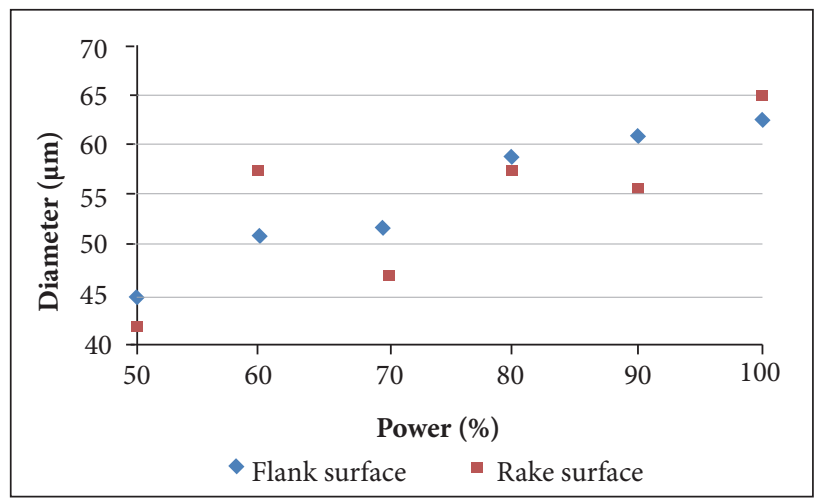

Figure 4. Diameter's behavior through power percentage variation.

Focus position is one of the most critical process parameters and directly affects the remaining geometry after material removal (Bordatchev and Nikumb, 2006).

On both tool surfaces, the decrease in laser focus distance caused an increase in the spot's diameter, which was the result expected according to the situation presented in Fig. 3.

Figures 6 and 7 show the result of the laser surface treatment on both tool surfaces.

Althought increasing the power increases the diameter's values, it also causes other effects on the rings, as it can be seen in Figs. 6 and 7. The images show that the tests where the power percentage was higher presented less precision

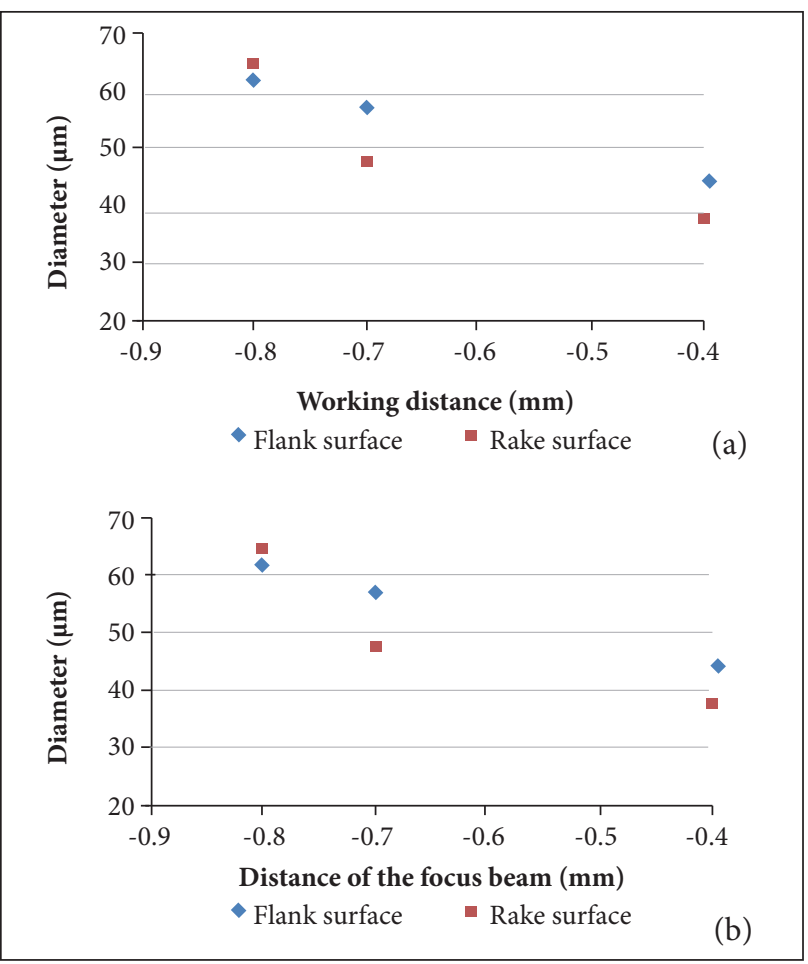

Figure 5. Diameter's behavior through working distance variation.

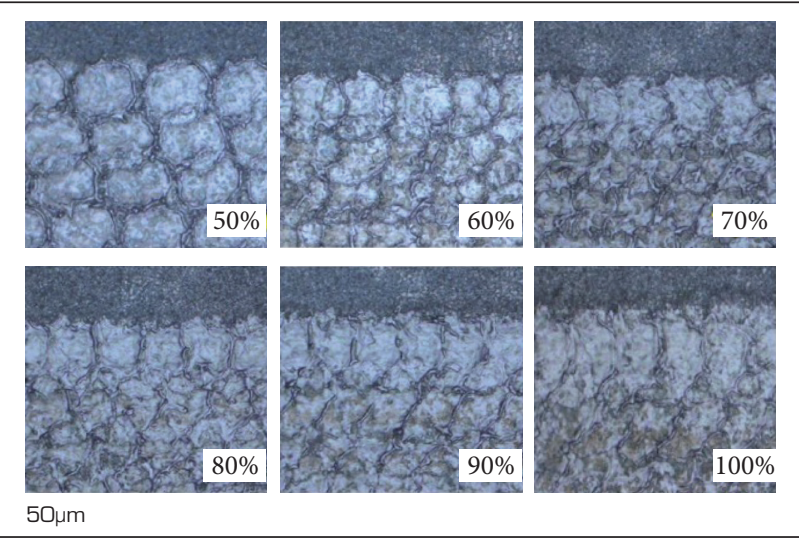

Figure 6. Images of ring's texture for different power percentage values on flank tool surface.

in the diameter's shape. In both surfaces, it is possible to notice that the laser ring's edges become less defined as the power grows.

This phenomenon can be explained by the texturing laser mechanism. Since the utilzed laser pulses where set to $100 \mathrm{~ns}$ in duration, the laser matter interaction time is long enough to enable a large amount of material being melted whithin the pulse duration. Evaporating material evolves a huge back pressure on the underlying material, which, in this case, is in melted state and thus can be expelled to the outer region of the irradiation zone. 


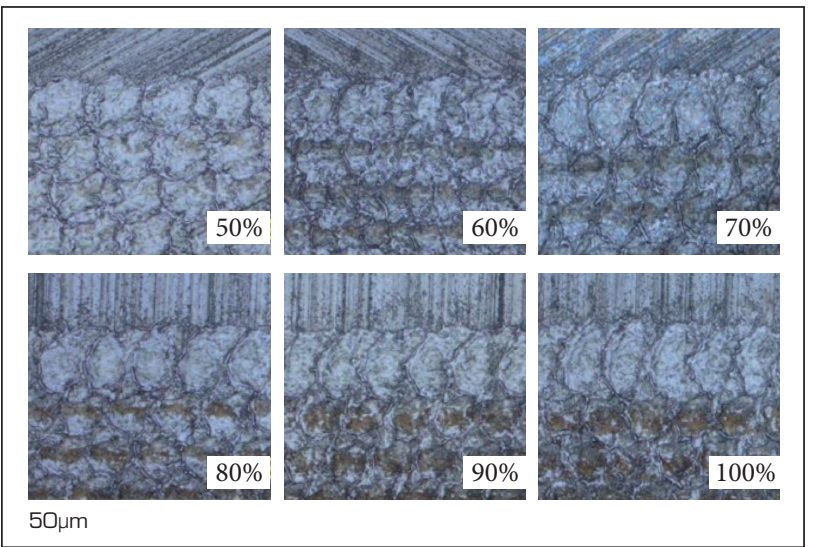

Figure 7. Images of ring's texture for different power percentage values on rank tool surface.

\section{DEPTH ANALYSIS}

The second microstructure parameter analyzed was the depth of the rings. For this analysis, the same variation of laser parameters used for diameter's analysis was used.

Figure 8 shows the dependency of the resulting ring's depth on laser's power. The working distance was kept constant at $0.8 \mathrm{~mm}$. Figure 8 also shows that, for the flank surface, there is a slight decrease in depth when the power increases. It is also possible to see, for the rake surface, that the depth slightly increases as well, when the power percentage increases.

In previous researches, Dumitru et al. (2004) presented a study where one could observe that higher incident fluencies generated larger ablation rate. In other words, it means that with/ when increasing the laser power, the depth will also increase.

However, the same behavior is not seen in either the studied cases. Changing the power in the rings' formation does not present the same correlation as in the pure ablation process, when analyzing the depth of the microstructure.

Caused by the formation principle, described before, the resulting surface after the meltiwng is not flat, but with peaks and valleys, created by the solidification of the thermally driven motion of molten material. This surface characteristic makes it difficult to define a right spot to measure the depth. In addition, the depth profile of the rings is just slightly above the order of the original surface's roughness, which also complicates the depth's determination.

For the second analysis of the microstructure's depth, the power was kept constant at $100 \%$, while the working distance was varied. The graph from Fig. 9 shows a comparison of the depth behavior between the two tested surfaces. In the rake surface, it is seen that when increasing the working distance, the depth

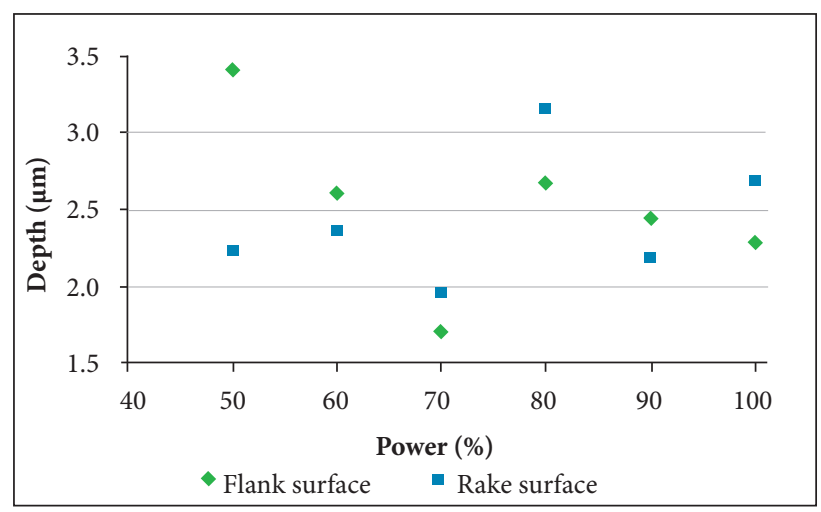

Figure 8. Graph of depth's behavior through power variation on the flank and rake tool surfaces.

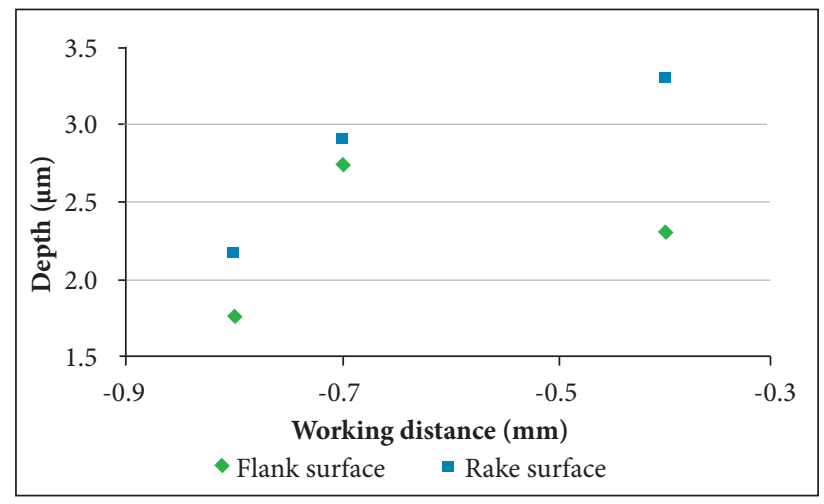

Figure 9. Ablated depth for different fluencies.

also decreases. In the flank surface, however, this behavior is not constant, although, in general, there is the decrease of the depth with the increase of the working distance.

It is known that, when the laser beam operates in its exact focus, the incident intensity (and also fluency) in the irradiated surface's area is maximal. For a pure ablation processes, the bigger the incident intensity, the bigger is the ablation rate during the process, which leads to a bigger depth. However, the intensity hitting the surface is decreased when the laser beam works out of its focus. Thus, the manufacturing of the ring's texture needs to be conducted in a defocused range in order to find the optimum intensity. The latter is found when the amount of evaporated material is just sufficient to force the molten material to form a ring exhibiting the desired shape.

\section{CONCLUSION}

Based on this investigation, the following conclusions could be drawn: 
- Increasing the laser power or the distance of the laser beam focus from the surface led to an increase in the rings' diameter on the flank and rake surface due to enhanced thermal affection of the surface's material. These results are in agreement with the results for the laser ablation process.

- The variation in the incident power beam generated different results in the microstructure's depth of each tested surface. This difference was explained by the process of ring formation and by the superficial melting in the surface's material caused by the laser. The behavior of the ring's structural formation is not the same as the phenomenon of ablation.

- The variation in the working distance caused the same result in depth of both studied surfaces. Increasing the working distance caused the decrease on the ring's depth, which corresponds to the results of the laser ablation process.

Further studies are required to evaluate the tribological performance of such ring's textures on the desired application.

\section{ACKNOWLEDGMENTS}

This work was supported by the Deutsche Forschungsgemeinschaft (German Research Foundation) and Coordenação de Aperfeiçoamento de Pessoal de Nível Superior (CAPES), within the research project "Surface conditioning and texturing of cutting tools edges for the optimization of manufacturing processes - ConTexTool”.

\section{REFERENCES}

Aurich, J.C., Zimmermann, M. and Leitz, L., 2011, "The Preparation of Cutting Edges Using a Marking Laser", Production Engineer, Vol. 5, No.1, pp. 17-24. doi: 10.1007/s11740-010-0275-9

Bordatchev, E. and Nikumb, S.K., 2006, "Effect of Focus Position on Informational Properties of Acoustic Emission Generated by LaserMaterial Interactions", Applied Surface Science, Vol. 253, No. 3, pp. 1122-1129. doi: 10.1016/j.apsusc.2006.01.047

Cabeza, M., Castro, G., Merino, P., Pena, G. and Román, M., 2012, "Laser Surface Melting: a Suitable Technique to Repair Damaged Surfaces Made in 14 Ni (200 grade) Maraging Steel", Surface and Coatings Technology, Vol. 212, pp. 159-168. doi:10.1016/j.surfcoat.2012.09.039

Dumitru, G., Romano, V., Weber, H.P., Gerbig, Y., Haefke, H., Bruneau, S., Hermann, J. and Sentis, M., 2004, "Femtosecond Laser Ablation of Cemented Cabides: Properties and Tribological Applications", Applied Physics A - Materials Science \& Processing, Vol. 79, No. 3, pp. 629-632. doi: 10.1007/s00339-004-2675-1

Etsion, I., 2004, "Improving Tribological Performance of Mechanical Components by Laser Surface Texturing", Tribology Letters, Vol. 17, No. 4, pp. 733-737. doi: 10.1007/s11249-004-8081-1

Janssen, A., 2012, "Laserstrukturieren senkt Reibung hochbelasteter Serienteile", Maschinenmarkt, Vol. 11.

Kim, B.S., Chung, W.Y., Rhee, M. and Lee, S., 2012, "Studies on the Application of Laser Surface Texturing to Improve the Tribological Performance of AICrSiN - Coated Surfaces", Metals and Materials
International, Vol. 18, No. 6, pp. 1023-1027. doi: 10.1007/ s12540-012-0483-2

Klocke, F., Gierlings, S., Adams, O., Aerbach, T., Kamps, S., Veselovac, D., Eckstein, M., Kirchheim, A., Blattner, M., Thiel, R. and Kohler, D., 2012, "New Concepts of force measurement systems for specific machining processes in aeronautic industry", Proceedings of the 5th Conference on High Performance Cutting.

Klocke, F. and Schmitt, R., 2012, "Surface conditioning and texturing of cutting tools for the optmization of machining processes". Proceedings of the 3rd International Meeting of Brazilian-German Collaborative Research Initiative on Production (BRAGECRIM), Aachen, Germany.

Meijer, J., Du, K., Gillner, A., Hoffmannc, D., Kovalenkod, V.S., Masuzawae, T., Ostendorfc, A., Poprawec, R. and Schulzc, W., 2002, "Laser Machining by Short and Ultrashort Pulses, State of Art and New Opportunities in the Age of the Photons", CIRP Annals - Manufacturing Technology, Vol. 51, No. 2, pp. 531-550. doi:10.1016/S0007-8506(07)61699-0

Poprawe, R., 2004, "Lasertechnik für die Fertigung", Springer, Heidelberg, Germany, 526p.

Scaraggi, M., Mezzapesa, F.P., Carbone, G., Ancona, A. and Tricarico, L., 2013, "Friction Properties of Lubricated Laser-MicrotexturedSurfaces: An Experimental Study from Boundary- to HydrodynamicLubrication", Tribology Letters, Vol. 49, No. 1, pp. 117-125. doi: 10.1007/s11249-012-0045-2 